\title{
Utilidad de la tecnología de reconocimiento automático de expresión facial aplicada al análisis de conducta criminal. Un estudio de caso.
}

Utility of automatic facial expression recognition technology applied to criminal behavior analysis. A case study.

\author{
Sergio Martínez Pacheco, Ph.D. ${ }^{1}$ \\ Rafael Manuel López Pérez, Ph.D. ${ }^{2}$ \\ Juan Enrique Soto Castro, Ph.D ${ }^{1}$ \\ Anderson Tamborim ${ }^{3}$ \\ Alicia Juárez Bielsa ${ }^{1}$
}

(1)Policía Nacional. Sección Análisis de Conducta (SAC), Madrid, España.

(2) Fundación Universitaria Behavior \& Law, Madrid, España.

(3) Social Intelligence Group, Sao Paulo, Brasil.

Email correspondencia: copj.ucic22@policia.es

\begin{abstract}
Resumen
Con el presente estudio se trata de validar la incorporación del uso de las nuevas tecnologías, especializadas en el reconocimiento de la expresión facial, al ámbito del estudio de la conducta criminal. Fruto de la colaboración de dos mundos distintos pero totalmente complementarios como son el campo académicoinvestigador y el campo aplicado, surge la posibilidad de implementar el uso de un software de codificación facial que automatiza la discriminación de las denominadas unidades de acción en el rostro de las personas a un caso concreto seleccionado por la unidad policial encargada de realizar estudios de comportamiento no verbal en el ámbito de la Policia Nacional, la Sección de Análisis de Conducta (SAC). Tras la confrontación de los resultados extraídos por los analistas de la SAC frente a los resultados arrojados por el software de reconocimiento facial, se llega a la conclusión de que la misma puede servir de gran utilidad de cara a facilitar la tarea de discriminación, si bien dicho análisis final debe ser suplementando por la experiencia y la supervisión de los analistas asignados al caso, puesto que los mejores resultados finales se obtienen del trabajo combinado de ambos.
\end{abstract}

Palabras Clave: comportamiento no verbal, expresión facial, software de reconocimiento, estudio comparativo, investigación policial.

\begin{abstract}
This study aims to validate the incorporation of the use of new technologies, specialized in the recognition of facial expression, into the study of criminal behavior. As a result of the collaboration of two different but totally complementary worlds, such as the academic-research field and the applied field, the possibility of implementing the use of facial coding software has arisen. This program automates the discrimination of the so-called units of action in the face of people to a specific case selected by the police unit in charge of non-verbal behavior studies in the National Police, the Behavior Analysis Section (S AC). After the confrontation of the results obtained by the analysts of the SAC with the results produced by the facial recognition software, it is concluded that it can be very useful to facilitate the task of discrimination, although this final analysis should be supplemented by the experience and supervision of the analysts assigned to the case, since the best final results are obtained from the combined work of both.
\end{abstract}

Keywords: nonverbal behavior, facial expression, recognition software, comparative study, police investigation.

Fecha de envío: 04/06/2018

Fecha de aceptación: 24/11/2018 


\section{INTRODUCCIÓN}

El análisis del comportamiento no verbal intenta vislumbrar lo que una persona piensa, le motiva y siente a través de los tres procesos básicos psicológicos de mayor interés: la cognición, la motivación y la emoción (López, Gordillo, \& Grau, 2016). La presente investigación se centra en el último de ellos, en un intento de analizar qué nos dicen las emociones sobre lo que estamos sintiendo, si existen maneras de escudriñar exteriormente, desde un observador ajeno a la situación, dichas emociones, y si las mismas pueden ser igualmente divisadas por un analista entrenado y/o por un software especializado.

Como recogen Fernández-Abascal, Jiménez \& Martín (2003), las emociones se entienden como un "proceso que implica una serie de condiciones desencadenantes (estimulos relevantes), la existencia de experiencias subjetivas o sentimientos (interpretación subjetiva), diversos niveles de procesamiento cognitivo (procesos valorativos), cambios fisiológicos (activación), y patrones expresivos y de comunicación (expresión emocional) que tiene unos efectos motivadores (movilización para la acción) y una finalidad: la adaptación a un entorno en continuo cambio"(p.50).

Respecto a esos patrones expresivos de comunicación, los mismos juegan un papel fundamental, en cumplimiento de su función social, en cuanto que permiten tanto la transmisión de las emociones y sentimientos a otros interlocutores, como, por otro lado, ser capaces de reconocerlos, todo ello sin necesidad de desarrollar capacidades extra por encima de las que traemos de serie ${ }^{1}$.

A partir de nuestra percepción, la interpretación del mundo en general, y el reconocimiento de emociones en particular, está determinada por la interacción entre la estructura biológica del cerebro y la experiencia que modifica dicha estructura, si bien existen diferencias en cuánto a determinar quién juega el papel fundamental. Así, existen defensores de factores neurológicos, que hablarían de circuitos especializados para observar y comprender los movimientos de los seres que nos rodean que traeríamos de serie en nuestro cerebro (Calvo-Merino, Grèzes, Glaser, Passingham, \& Haggard, 2006), encontrándose a su vez diferencias de género basadas en el diferente desarrollo

${ }^{1}$ Personas sin un entrenamiento específico pueden reconocer las emociones de sus interlocutores a través de las expresiones faciales de éstas (Hager \& Ekman, 1983). psicológico de hombres y mujeres (Babchuck, Hames, \& Thompson, 1985), pasando por los defensores del contexto como generador de emociones y, por tanto, en su expresión (Mallo, Fernández, \& Wallbott, 1989), hasta llegar a la diferenciación intra-emocional en cuanto al reconocimiento mejor o peor de una emoción en función de la valencia o el tono hedónico y la activación de la misma, de tal manera que emociones positivas con un nivel alto de activación (como por ejemplo la sorpresa y el miedo, respectivamente) se reconocerían fácilmente bajo una exposición relativamente breve a los estímulos (Ogawa \& Suzuki, 1999).

Por otro lado, desde el ámbito de la expresión emocional se pueden diferenciar varias etapas: una primera, con Darwin (1872) como máximo representante, que hablaría de que los patrones de respuesta expresiva emocionales son innatos y determinado por programas genéticos (Chóliz \& Tejero, 1994); una segunda etapa en la primera mitad del siglo XX, protagonizada por Boring, Floyd, Allport, Tichener y Woodworth (Chóliz \& Tejero, 1994) desautorizando la universalidad de las expresiones faciales de las emociones básicas (Russelll, 1994); una tercera etapa que duraría desde 1940 a 1960, protagonizada por una sequía investigadora, destacando investigaciones como las de Schlosberg $(1952,1954)$. Y finalmente, aparecería lo que se podría denominar como la cuarta etapa, que marcaría el segundo gran hito en el estudio de esta materia: las aportaciones de Ekman y Friessen.

Dentro de los múltiples canales de expresión de la emociones, la expresión facial ha sido probablemente el canal expresivo más presente en la investigación científica, considerada a su vez como la principal vía de comunicación de las experiencias emocionales voluntarias y espontáneas, jugando un papel fundamental en las interacciones sociales por tener una influencia de doble vía, por un lado en cuanto a la capacidad de modular el nivel de intensidad generado en el emisor, y por otro, el contagiar dicha emoción en el receptor, convirtiéndose en un poderoso transmisor de los sentimientos (Frith, 2009).

La importancia que desde el ámbito científico se le ha otorgado a la expresión facial de las emociones se ha visto reflejada en la propia evolución de las investigaciones llevadas a cabo. Comenzando por los años setenta con numerosos estudios (RW Buck, Savin, \& Miller, 1972; Ekman \& Friesen, 1971; Tourangeau \& Ellsworth, 1979; Zuckerman, Hall, DeFrank, \& Rosenthal, 1976), pasando por los años ochenta que profundiza en diferentes aspectos 
como la asimetría del movimiento facial estudiada en el campo de la detección de mentiras en cuanto a discernir entre expresiones espontáneas de las deliberadas (Ekman, 1980; Ekman, Hager, \& Friesen, 1981), estudios relacionales entre dicha asimetría con la implicación diferencial de los hemisferios cerebrales en el procesamiento emocional (Borod, Caron, \& Koff, 1981; Bruyer, 1981; R. Buck, 1980; Moscovitch \& Olds, 1982), la inhibición social de la expresión facial como represión del comportamiento en presencia de otras personas (Yarczower \& Daruns, 1982), implicaciones en el estudio del dolor (Craig \& Patrick, 1985; LeResche \& Dworkin, 1984), la teoría del feedback facial (Duclos et al., 1989), diferentes usos en el campo de la influencia y atractivo hacia los demás (Mueser, Grau, Sussman, \& Rosen, 1984; Mullen et al., 1986), hasta finalmente llegar a la década de los años noventa hasta nuestros días, donde se han estudiados los déficits en la percepción y expresión de las emociones (Cornellá \& Llusent, 2003; Rourke, 1995; Torgeson, 1993), nuevos avales a la teoría del feedback facial (Hennenlotter et al., 2009; Mojzisch et al., 2006; Schilbach et al., 2006; Schilbach, Eickhoff, Mojzisch, \& Vogeley, 2008; Schilbach, Koubeissi, David, Vogeley, \& Ritzl, 2007) interpretando la expresión de las emociones como un potenciador de las mismas (si nos enfadamos y ponemos cara de enfado, estaremos más enfadaos que si nos enfadamos pero evitamos poner cara de enfado), así como las inferencias realizadas, erróneas o no, de rasgos de personalidad a través de la expresión facial (Bar, Neta, \& Linz, 2006; Knutson, 1996), inclusive en campos aplicados como en los juicios elaborados por los votante sobre la personalidad de los políticos (Olivola \& Todorov, 2010).

La creación de sistemas de codificación específicos para medir expresiones faciales ha supuesto un punto de partida para infinidad de nuevas líneas de investigación. Igualmente dichos sistemas han posibilitado la clasificación de la expresión facial en función del punto de vista subyacente en cada uno de los mismos.

De este modo, podemos hablar de sistemas como el FACS (Ekman \& Friesen, 1978), FAST (Ekman, Friesen, \& Tomkins, 1971), EMFACS (Friesen \& Ekman, 1983), FACSAID (Hager, 2003), y MAX (Izard, 1979), donde las emociones son consideradas como entidades discretas independientes las unas de las otras, o bien conformantes de una dimensión global en la cual se situarían todas ellas, destacando autores de este enfoque categorial como Darwin (1872), Tomkins (1962) con su teoría del feedback facial, Ekman y Friesen (1978), e Izard (1979). Por contra, otros sistemas, como el sistema FACES (Kring \& Sloan, 2007), se situarían en concepciones del enfoque dimensional, donde las emociones se posicionan en un punto de un continuo determinado por aspectos como la valencia (entre placer y displacer), arousal o activación (entre activación y desactivación) y control (entre situación sentida como totalmente controlada por el sujeto y situación sentida como totalmente incontrolada por el sujeto).

Si bien los anteriores sistemas de codificación han posibilitado el desarrollo de la investigación, no es un campo totalmente abarcado por los mismos, sino que es necesario analizar detenidamente nuevos sistemas de codificación basados en programas informáticos que son capaces de reconocer la expresión facial utilizando algoritmos y diversos inputs tales como imágenes a través de cámaras web o grabaciones de vídeo procedentes de archivos audiovisuales que ayuden a mejorar la precisión y eficiencia de los análisis realizados en las expresiones faciales (Lewinski, den Uyl, \& Butler, 2014).

Existen estudios llevados a cabo con el software utilizado en el presente estudio, FaceReader (Noldus, 2014), en diferentes campos del conocimiento como la Psicología Social (Chentsova-Dutton \& Tsai, 2010), las Ciencias del Comportamiento del Consumidor (Chan, Boven, \& Andrade, 2014), o el mundo publicitario (Lewinski, Fransen, \& Tan, 2014), donde se ha testado el uso de programas informáticos para el estudio de la expresión facial de las emociones, pero no existen tantas investigaciones en el ámbito criminológico, por lo que el presente artículo quiere contribuir a la validación de dichas herramientas como ayuda futura (y por qué no, inmediata) para los analistas de las fuerzas y cuerpos de seguridad.

Se ha considerado importante desde el ámbito policial (Magariños, 2015) el promover investigaciones sobre comportamientos no verbales a través de proyectos de colaboración académica que puedan materializarse en el desarrollo de estudios de campo que consigan implementar los resultados obtenidos en la metodología ya existente por las unidades especializadas policiales, ya que, en contra de la creencia popular de que los policías poseen una capacidad especial para detectar mentiras, ningún estudio ha demostrado que los agentes sean mejores que cualquier persona de la calle a la hora de distinguir a los interlocutores mentirosos de los sinceros (Akehurst, Köhnken, Vrij, \& Bull, 1996; de Paulo \& Pfeifer, 1986; 
Garrido, Masip, \& Herrero, 2004; Vrij, 2008).

Por tanto, son diferentes los campos del conocimiento donde se ha testado la herramienta informática FaceReader (FR) para la codificación de la expresión facial. Igualmente se ha reflejado la importancia de la colaboración entre el mundo académico y el mundo profesional. Por todo ello, se considera de interés plantear el siguiente estudio de caso, teniendo en cuenta que los resultados y conclusiones que se obtengan pueden repercutir de manera directa en el trabajo diario de los analistas de conducta criminal.

\section{MÉTODO}

La hipótesis de partida será que el análisis realizado con la herramienta informática de codificación facial coincidirá con el análisis realizado por los expertos de la Sección de Análisis de la Conducta de la Policía Nacional.

\section{A. Material}

Para la presente investigación se ha recurrido al estudio del comportamiento no verbal llevado a cabo por analistas de la Sección de Análisis de Conducta (SAC) sobre una video-reconstrucción judicial hecha en el año 2015, con una duración total de seis minutos y veinticinco segundos.

En dicha reconstrucción la persona protagonista en las imágenes narra la realización de un robo violento y posterior asesinato $^{2}$ de una turista que se encontraba realizando el Camino de Santiago, tras confesar ser el autor de los hechos en presencia de la Comisión Judicial, por el cual ha sido condenado posteriormente en Juicio Oral a la suma de 23 años de prisión.

Los analistas de la SAC llevaron a cabo el estudio mediante una herramienta de trabajo denominada protocolo NBAM³ (López, Gordillo, \& Soto, 2016). Dicho protocolo se basa en una matriz en la cual se cruzan diferentes fuentes de datos que se van estructurando a lo largo de cinco niveles, numerados desde el nivel 0 hasta el nivel 4 . Para la presente investigación sólo se tuvo en cuenta la fase del nivel 1, que se corresponde con aquellos datos ${ }^{4}$ que

\footnotetext{
2 Según Sentencia 00167/2017 de 11 de abril de 2017 de la Audiencia Provincial de León - Tribunal del Jurado.

${ }^{3}$ Nonverbal Behavior Analysis Matrix

${ }^{4}$ En la matriz se contempla el registro de múltiples datos procedentes de diversos canales como: expresión facial (EF); gestos $(\mathrm{G})$; posturas, orientación y movimiento $(\mathrm{P})$; paralenguaje $(\mathrm{PA})$; proxémica $(\mathrm{PR})$; háptica $(\mathrm{H})$; apariencia $(\mathrm{A})$; oculésica (O); y verbal (V).
}

solo hacen referencia a la codificación de la expresión facial, puesto que es la que se quiere comparar con los análisis realizados por el FaceReader.

Cabe recordar que acorde al nivel del protocolo NBAM en el cual había que centrarse para el presente estudio (Nivel 1: Datos) en ningún momento se incluyeron opiniones sobre lo observado, sino única y estrictamente datos referentes a la expresión facial registrada en las imágenes, puesto que el objetivo principal era enfrentar los datos recogidos por analistas de la SAC frente a los codificados por el software específico.

Los datos que se registran sobre expresión facial son las denominadas Unidades de Acción (UA), que son realizadas por uno o varios músculos que están en contracción o relajación (Fernández-Abascal \& Chóliz, 2001). Este sistema puede codificar prácticamente cualquier expresión facial anatómicamente posible a través de su deconstrucción en unidades específicas de acción y sus segmentos temporales. Las UAs son independientes de cualquier interpretación, lo cual proporciona al sistema una alta utilidad para el ámbito científico.

En la siguiente Tabla 1 se observa cómo se realizó el estudio por parte de los analistas con ayuda de la matriz del protocolo NBAM. Cada fotograma a estudio iba generando una nueva fila de datos, especificando siempre la verbalización asociada al mismo, y una descripción de la conducta desgranada en forma de unidades de acción.

Tabla 1. Tabla de Datos del Protocolo NBAM

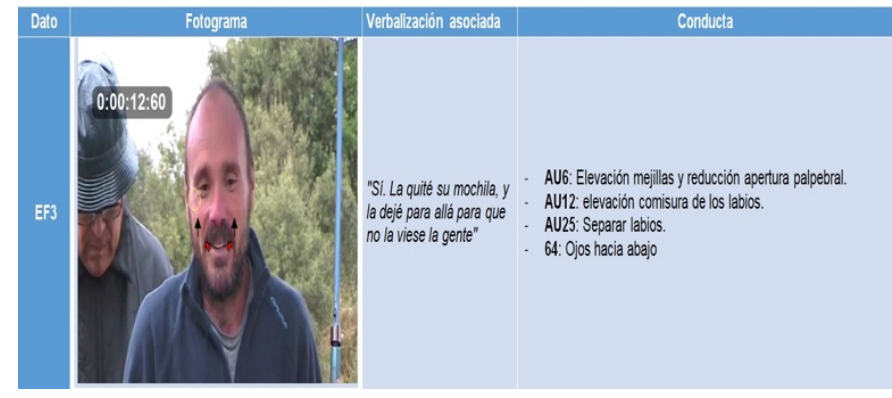

Por su parte el análisis realizado por ordenador se ha realizado con el software informático FaceReader (Noldus, 2014), en su séptima versión, el cual resulta ser un programa informático de reconocimiento automático de emociones basado en el análisis de expresiones faciales.

En investigaciones previas ya se avanzaba hasta un $89 \%$ en precisión de reconocimiento en las seis emociones básicas (den Uyl \& van Kuilenburg, 2005), aspecto igualmente revalidado en posteriores estudios (Lewinski, 
den Uyl, et al., 2014), valores que se pueden ajustar bastante bien a la capacidad de precisión que muestran personas analistas en el reconocimiento de emociones en la expresión facial (Nelson \& Russell, 2013; Russelll, 1994).

Dicho software trabaja en tres fases, comenzando en la detección del rostro de la persona que se va a evaluar, identificando posteriormente unos puntos clave a lo largo del rostro (Figura 1) que contrasta con los de un modelo tridimensional (Cootes \& Taylor, 2004), y por último clasificando la imagen acorde a la aproximación entre la misma y la emoción presentada. El software puede dar un triple resultado de clasificación como, una emoción propiamente dicha de entre las seis emociones básicas, una cara neutra, o como una imagen en la cual no se ha podido identificar el rostro (e.g. porque la cara de la persona a evaluar se encuentre ladeada con cierto ángulo respecto al plano de imagen desde el cual se está grabando).

Figura 1: Fotograma del análisis con FaceReader

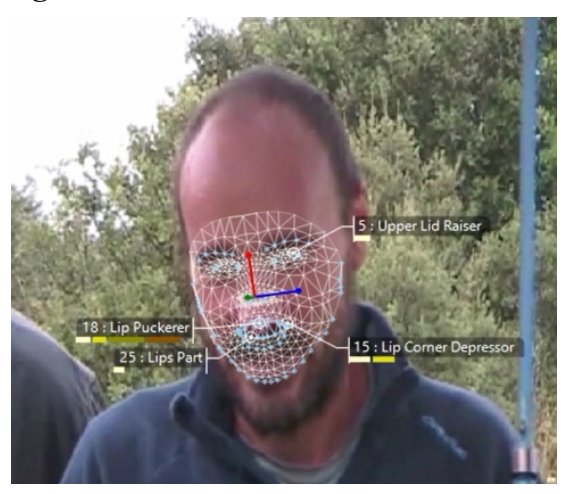

Igualmente la herramienta informática evalúa a cada imagen con rangos numéricos de 0 a 1 , significando aquellos valores más altos (los próximos a la unidad) la mayor probabilidad de estar experimentando la emoción etiquetada, y viceversa, bajas puntuaciones estarían indicando una baja probabilidad de que dicha emoción estuviera siendo sentida por la persona objeto del análisis.

\section{B. Procedimiento}

En primer lugar, para la composición de la matriz del protocolo NBAM se procedió a una selección de aquellos fotogramas más relevantes, según criterio de los analistas de la SAC, en base a aquellos momentos donde resultaba más interesante observar si lo verbalizado por el protagonista de las imágenes resultaba congruente o no con lo expresado a nivel facial, esto es, seleccionado aquellos tramos de vídeo más conflictivos y convenientes de clarificar en un hipotético informe de comportamiento no verbal solicitado por el grupo investigador del caso o la propia autoridad judicial.

Una vez extraídos dichos fotogramas, los mismos fueron codificados en clave de unidades de acción faciales por un primer analista asignado al caso. Posteriormente, dichos resultados fueron supervisados por un segundo especialista en la realización de informes sobre comportamiento no verbal.

En segundo lugar, el analista encargado de la codificación facial informatizada realizó la misma con el FaceReader, destacando aquellas partes donde el rostro de la persona objeto de estudio era discriminado y analizado.

Finalmente, la matriz resultante fruto del acuerdo interjueces de los analistas de la SAC, fue contrastada con la codificación arrojada por el FR.

\section{RESULTADOS}

A la vista de los resultados que se obtuvieron, los mismos se encuadraron en dos grandes bloques para una mejor interpretación de los mismos a posteriori.

Por un lado, tenemos fotogramas donde los analistas codificaban unidades de acción mientras que el FR no lo hacía (Tabla 2), y otros casos donde el proceso era a la inversa, esto es, el programa informático codificaba la expresión facial mientras que los analistas no consideraron dicho fotograma relevante como objeto de estudio.

Tabla 2: Matriz de discrepancia Analista vs FR

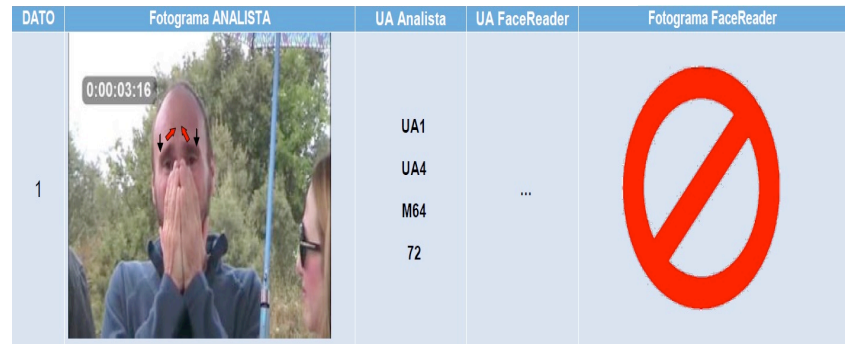

Y por otro lado, tenemos otro gran bloque donde tanto analistas como FR coincidían en la selección del fotograma objeto de estudio. Para ello, se construyó una matriz, denominada matriz de discrepancias, donde se pusieron en común los resultados de los analistas y del FR.

En esta matriz, los resultados fueron igualmente dispares, observándose como tónica general que el FR discriminaba más cantidad de unidades de acción que los analistas, especialmente en aquellos fotogramas donde la calidad de la imagen no era buena. 


\section{CONCLUSIONES}

Teniendo en cuenta la hipótesis de partida de que los análisis realizados con la herramienta FaceReader coincidirían en su mayor parte con los hechos por los expertos de la SAC, cabe decir a la luz de los resultados, que dicho software se muestra, en aquellos segmentos del vídeo donde coinciden las codificaciones de los analistas con las del FR, como una inestimable herramienta de ayuda para el analista de la codificación facial, dada su mayor capacidad de discriminación de unidades de acción.

Si bien cabría hacer un matiz, puesto que no siempre cantidad es sinónimo de calidad en el estudio, esto es, se observó que a veces la disparidad en cuanto al número de unidades de acción codificadas entre analistas y software era debido a que los primeros habían tenido en cuenta durante todo el estudio el establecimiento de una línea base del rostro del protagonista. Esto hizo que muchas veces los analistas remarcaran unidades de acción que bien no eran propias del rostro del protagonista, o inclusive formando parte de la línea base del mismo, la intensidad en la expresión de las mismas era digna de ser reflejada.

Junto a lo anterior hay que tener presentes una serie de limitaciones técnicas que se encontraron y que debieran ser tenidas en cuenta en estudios futuros, así como en las sucesivas versiones del software de cara a mejorar la potencialidad del mismo (Figura 2). Entre dichas limitaciones, destacan la necesidad de delimitar un tipo de plano específico donde el programa informático realice el análisis de forma continuada y sin interrupciones, ya que en el caso de estudio no siempre se analizaba la imagen independientemente del tipo de plano que se estuviera filmando. Fruto de lo anterior, se ven fotogramas de plano frontal donde se ve el rostro con total nitidez pero donde no hay codificación del FR (1), y otros donde la persona se encuentra ladeada y sin embargo si logra registrar unidades de acción (2). Otra dificultad añadida observada es la necesidad de mantener la imagen limpia de ruidos o contaminaciones en forma de otros rostros de personas que pudieran aparecer en imagen, ya que se encontraron fotogramas donde el programa pasaba a analizar solo el rostro de esa segunda persona que aparecía en imagen (3). Y finalmente, señalar fotogramas en los que el programa informático confundía lo que interpretaba como rostro con una visión lateral de la cara y resto del cuerpo, como por ejemplo identificar una supuesta barbilla con lo que finalmente resultaba ser un hombro (4).
Figura 2: Limitaciones encontradas

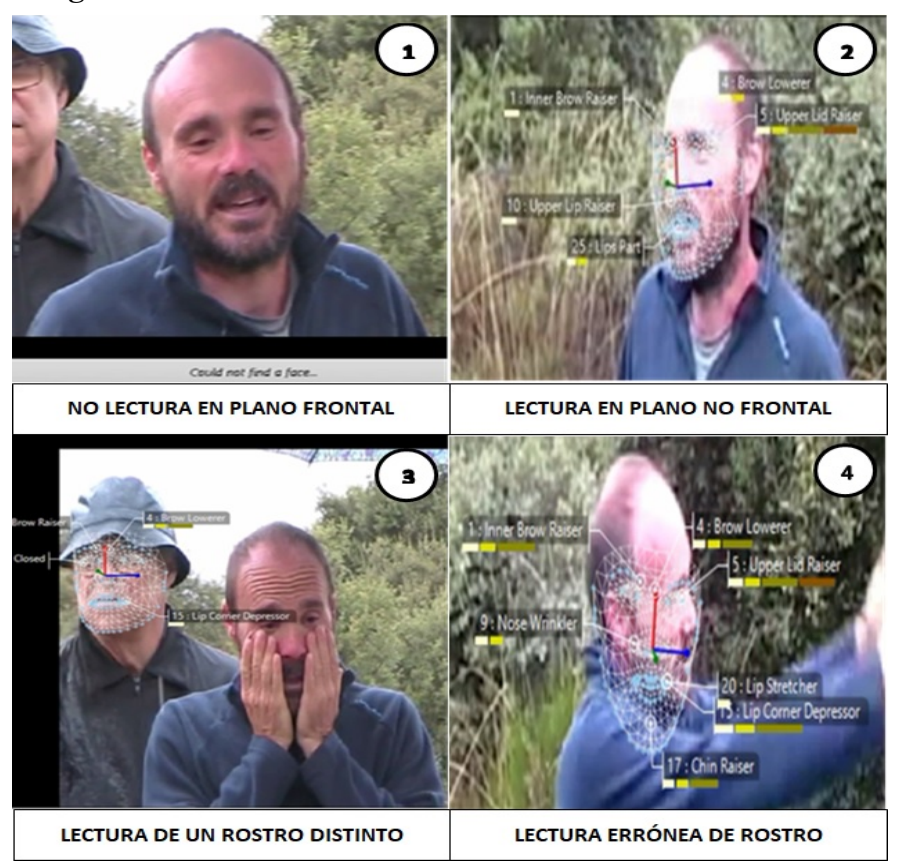

A la luz de estos resultados y conclusiones finales, cabe decir que es indudable la ayuda que una herramienta informática, como la analizada, puede aportar al analista experimentado en el estudio del comportamiento no verbal. De tal manera, que la incorporación a la praxis diaria del analista no sólo puede suponer, en el corto plazo, una indudable mejora en las codificaciones faciales, sino que en el largo plazo, con las mejoras incorporadas que solventen las limitaciones observadas, se piense como una herramienta que brinde al analista la oportunidad de depositar todos sus recursos en el proceso posterior clave para un buen informe criminal de comportamiento no verbal, el proceso de generación de inferencias e hipótesis, basado en la previa codificación facial en unidades de acción, más susceptible de automatización.

Una prueba de la importancia de los estudios de comportamiento no verbal en el ámbito policial es su demanda desde áreas tan diversas como la policía de fronteras, seguridad ciudadana, y como no, en investigaciones judiciales sobre delitos contra la integridad física de las personas (homicidios, lesiones, etc), donde puede ayudar no solo en cuanto la preparación de interrogatorios o testimonios en juicios, sino también para mejorar la asistencia a las víctimas favoreciendo el rapport policial con las mismas.

Por lo que finalmente, no se aboga por una visión sustitutoria del trabajo humano por la herramienta informática, sino por la complementariedad de ambos. 


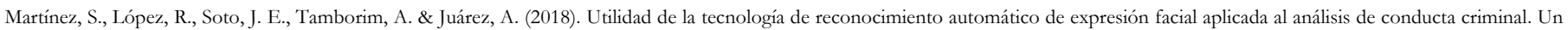
estudio de caso. Behavior \& Law Journal, 4(1), 1-8.

\section{REFERENCIAS}

Akehurst, L., Köhnken, G., Vrij, A., \& Bull, R. (1996). Lay persons' and police officers' beliefs regarding deceptive behaviour. Applied Cognitive Psychology, 10.

Babchuck, W. A., Hames, R. B., \& Thompson, R. A. (1985). Sex differences in the recognition of infant facial expressions of emotion: The primary caretaker hypothesis. Ethology and Sociobiology, 6(2), 89-101.

Bar, M., Neta, M., \& Linz, H. (2006). Very first impressions. Emotion, 6(2), 269-278.

Borod, J. C., Caron, H. S., \& Koff, E. (1981). Asymmetry in positive and negative facial expressions: Sex differences. Neuropsychologia, 19(6), 819-824.

Bruyer, R. (1981). Asymmetry of facial expression in brain damaged subjects. Neuropsychologia, 19(4), 615-624.

Buck, R. (1980). Nonverbal behavior and the theory of emotion: The facial feedback hypothesis. Journal of Personality and Social Psychology, 38(5), 811-824.

Buck, R., Savin, V., \& Miller, R. (1972). Communication of affect through facial expressions in humans. Journal of Personality and Social Psychology, 23(3), 362-371.

Calvo-Merino, B., Grèzes, J., Glaser, D. E., Passingham, R. E., \& Haggard, P. (2006). Seeing or Doing? Influence of Visual and Motor Familiarity in Action Observation. Cereb Cortex, 16(19), 1905-1910.

Chan, C., Boven, L. Van, \& Andrade, E. (2014). Moral violations reduce oral consumption. Journal of Consumer Psychology, 24, 381386.

Chentsova-Dutton, Y., \& Tsai, J. (2010). Self-focused attention and emotional reactivity: the role of culture. Journal of Personality and Social Psychology, 98, 507-519.

Chóliz, M., \& Tejero, P. (1994). Neodarwinismo y antidarwinismo en la expresión de las emociones en la psicología actual. Revista de Historia de La Psicología, 89-94.

Cootes, T., \& Taylor, C. (2004). Statistical models of appearance for computer vision. Manchester: Imaging Science and Biomedical Engineering, University of Manchester.

Cornellá, J., \& Llusent, A. (2003). Trastorno de déficit de atención con o sin hiperacti-vidad. Pediatría Integral, 7(8), 571-582.

Craig, K. D., \& Patrick, C. J. (1985). Facial expression during induced pain. Journal of Personality and Social Psychology, 48(4), 1080-1091.

Darwin, C. (1872). The Expression of the Emotions in Man and Animals. Nueva York: Appleton and Company.

de Paulo, B. M., \& Pfeifer, R. L. (1986). On the job experience and skill at detecting deceptions. Journal of Applied Social Psychology, 16.

den Uyl, M. J., \& van Kuilenburg, H. (2005). The FaceReader: Online facial expression recognition. In P.J.J.Noldus, F.Grieco, L.W.S.Loijens, \& P.H.Zimmerman (Eds.), Proceedings of the 5th International Conference on Methods and Techniques in Behavioral Research, Measuring Behavior (pp. 589-590). Wageningen,the Netherlands: Noldus InformationTechnology.

Duclos, S. E., Laird, J. D., Schneider, E., Sexter, M., Stern, L., \& Van Lighten, O. (1989). Emotion-specific effects of facial expressions and postures on emotional experience. Journal of Personality and
Social Psychology, 57(1), 100-108.

Ekman, P. (1980). Asymmetry in facial expression. Science, 209(4458), 833-834.

Ekman, P., \& Friesen, W. (1971). Constants across cultures in the face and emotion. Journal of Personality and Social Psychology, 17(2), 124-129.

Ekman, P., \& Friesen, W. (1978). Facial action coding system: a technique for the measurement of facial movement. Palo Alto: Consulting Psychologists.

Ekman, P., Friesen, W., \& Tomkins, S. (1971). Facial affect scoring technique: A first validity study. Semiotica.

Ekman, P., Hager, J. C., \& Friesen, W. V. (1981). The symmetry of emotional and deliberate facial actions. Psychophysiology, 18(2), 101-106.

Fernández-Abascal, E. G., \& Chóliz, M. (2001). Expresión facial de la emoción. Madrid: UNED.

Fernández-Abascal, E. G., Jiménez, M. P., \& Martín, M. D. (2003). Emoción y motivación: la adaptación bumana. (p.50) (vol.1) Madrid: Ramón Areces.

Friesen, W., \& Ekman, P. (1983). EMFACS-7: Emotional facial action coding system. Unpublished Manuscript, University of California at San.

Frith, C. (2009). Role of facial expressions in social interactions. Philosophical Transactions of the Royal Society of London B: Biological Sciences, 364(1535), 3453-3458.

Garrido, E., Masip, J., \& Herrero, C. (2004). Police officers' credibility judgments: Accuracy and estimated ability. International Journal of Psychology, 39.

Hager, J. C. (2003). Human Face. 2013. Retrieved from http://www.face-and-

emotion.com/dataface/facsaid/description.jsp Facial Action Coding System Affect Interpretation Dictionary (FACSAID)

Hager, J. C., \& Ekman, P. (1983). The inner and Outer Meanings of Facial Expressions. Nueva York: The Guilford Press.

Hennenlotter, A., Dresel, C., Castrop, F., Ceballos-Baumann, A. O., Wohlschläger, A. M., \& Haslinger, B. (2009). The link between facial feedback and neural activity within central circuitries of emotion-New insights from Botulinum toxin-induced denervation of frown muscles. Cerebral Cortex, 19(3), 537-542.

Izard, C. E. (1979). The Maximally Discriminative Facial Movement Coding System (MAX). Newark, Delaware: University of Delaware, Instructional Resoruce Center.

Knutson, B. (1996). Facial expressions of emotion influence interpersonal trait inferences. Journal of Nonverbal Behavior, 20(3), 165-182.

Kring, A., \& Sloan, D. (2007). The Facial Expression Coding System (FACES): development, validation, and utility. Psychological Assessment, 19(2), 210-224.

LeResche, L., \& Dworkin, S. F. (1984). Facial expression accompanying pain. Social Science \& Medicine, 19(12), 1325-1330.

Lewinski, P., den Uyl, T. M., \& Butler, C. (2014). Automated facial coding: Validation of basic emotions and FACS AUs in FaceReader. Journal of Neuroscience, Psychology, and Economics, 7(4), 227. 


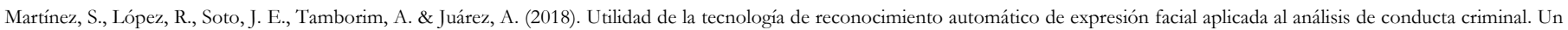
estudio de caso. Behavior \& Law Journal, 4(1), 1-8.

Lewinski, P., Fransen, M., \& Tan, E. (2014). Predicting advertising effectiveness by facial expressions in response to amusing persuasive stimuli. Journal of Neuroscience, Psychology, and Economics, 7, 1-14.

López, R. ., Gordillo, F., \& Soto, J. E. (2016). Protocolo NBAM (Nonverbal Behavior Analysis Matrix). In R. M. López, F. Gordillo, \& M. Grau (Eds.), Comportamiento no verbal (pp. 179192). Madrid: Ediciones Pirámide.

López, R. M., Gordillo, F., \& Grau, M. (2016). Comportamiento no verbal: más allá de la comunicación y el lenguaje. Madrid: Pirámide.

Magariños, J. G. (2015). El lenguaje del cuerpo: aplicación del análisis del comportamiento no verbal al ámbito policial. Ciencia Policial, 127, 7-62.

Mallo, M. J., Fernández, J. M., \& Wallbott, H. (1989). Reconocimiento de expresiones a partir de la expresión y el contexto: una réplica. Revista de Psicología Social, 4, 291-298.

Mojzisch, A., Schilbach, L., Helmert, J. R., Pannasch, S., Velichkovsky, B. M., \& Vogeley, K. (2006). The effects of selfinvolvement on attention, arousal, and facial expression during social interaction with virtual others: A psychophysiological study. Social Neuroscience, 1(3-4), 184-195.

Moscovitch, M., \& Olds, J. (1982). Asymmetries in spontaneous facial expressions and their possible relation to hemispheric specialization. Neuropsychologia, 20(1), 71-81.

Mueser, K. T., Grau, B. W., Sussman, S., \& Rosen, A. J. (1984). You're only as pretty as you feel: Facial expression as a determinant of physical attractiveness. Journal of Personality and Social Psychology, 46(2), 469-478.

Mullen, B., Futrell, D., Stairs, D., Tice, D. M., Baumeister, R. F., Dawson, K. E., ... Rosenfeld. (1986). Newcasters' facial expressions and voting behavior of viewers: Can a smile elect a president?. Journal of Personality and Social Psychology, 51(2), 291295.

Nelson, N. L., \& Russell, J. A. (2013). Universality revisited. Emotion Review, 5, 8-15. https://doi.org/10.1177/1754073912457227

Noldus. (2014). FaceReader: Tool for Automated Analysis of Facial Expression: Version6.0. Wageningen,the Netherlands: Noldus Information Technology B.V.

Ogawa, T., \& Suzuki, N. (1999). Response differentiation to facial expression of emotion as increising exposure duration. Perceptual and Motor Skills, 89(2), 557-563.

Olivola, C. Y., \& Todorov, A. (2010). Elected in 100 milliseconds: Appearance-based trait inferences and voting. Journal of Nonverbal
Behavior, 34(2), 83-110.

Rourke, B. P. (1995). Syndrome of nonverbal learning disabilities: Neurodevelopmental manifestations. Nueva York: Guilford Press.

Russelll, J. A. (1994). Is there universal recognition of emotion from facial expression?: A review of the cross-cultural studies. Psychological Bulletin, 115(1), 102-141.

Schilbach, L., Eickhoff, S. B., Mojzisch, A., \& Vogeley, K. (2008). What's in a smile? Neural correlates of facial embodiment during social interaction. Social Neuroscience, 3(1), 37-50.

Schilbach, L., Koubeissi, M. Z., David, N., Vogeley, K., \& Ritzl, E. K. (2007). Being with virtual others: studying social cognition in temporal lobe epilepsy. Epilepsy \& Behavior, 11(3), 316-323.

Schilbach, L., Wohlschlaeger, A. M., Kraemer, N. C., Newen, A., Shah, N. J., Fink, G. R., \& Vogeley, K. (2006). Being with virtual others: Neural correlates of social interaction. Neuropsychologia, 44(5).

Schlosberg, J. (1952). The description of facial expressions in terms of two dimensions. Journal of Experimental Psychology, 44(4), 229237.

Schlosberg, J. (1954). Three dimensions of emotion. Psychological Review, 61(2), 81-88.

Tomkins, S. (1962). Affect imagery consciousness: Volume I: The positive affects. Nueva York: Springer.

Torgeson, J. K. (1993). Variations on theory in learning disabilities. In G. R. Lyon, D. B. Gray, J. F. Kavanagh, \& N. A. Krasnegor (Eds.), Better understanding learning disabilities: New views from research and their implications for education and public policies (pp. 153-170). Baltimore, MD: Paul H. Brookes.

Tourangeau, R., \& Ellsworth, P. C. (1979). The role of facial response in the experience of emotion. Journal of Personality and Social Psychology, 37(9), 1519-1531.

Tribunal del Jurado - Audiencia Provincial de León (11 de abril de 2017). Sentencia 167/17 (2017).

Vrij, A. (2008). Detecting lies and deceit: Pitfalls and opportunities. John Wiley \& Sons, Ltd.

Yarczower, M., \& Daruns, L. (1982). Social inhibition of spontaneous facial expressions in children. Journal of Personality and Social Psychology, 43(4), 831-837.

Zuckerman, M., Hall, J. A., DeFrank, R. S., \& Rosenthal, R. (1976). Encoding and decoding of spontaneous and posed facial expressions. Journal of Personality and Social Psychology, 34(5), 966977. 